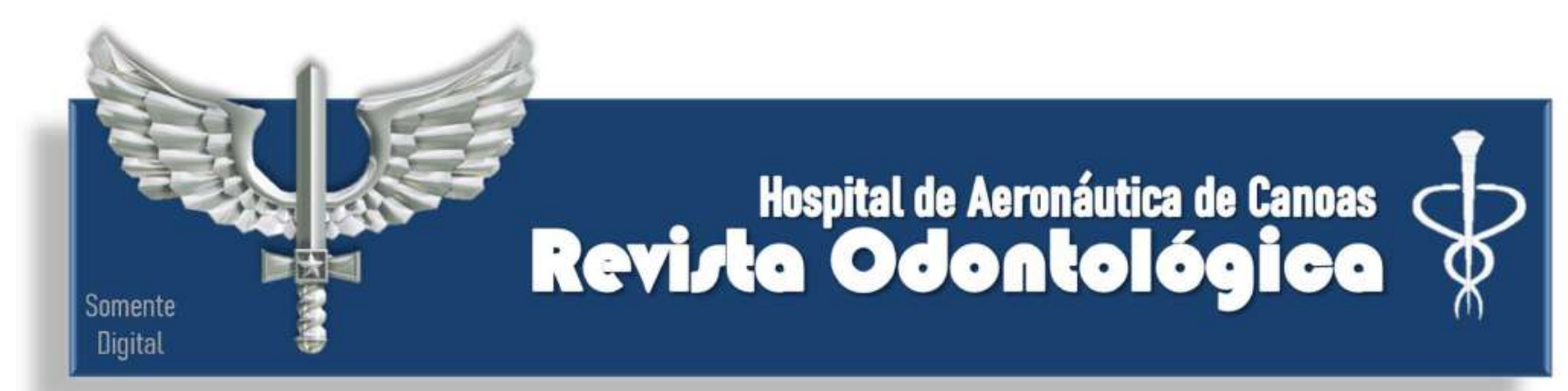

Relato de Caso Clínico

\title{
EMPREGO DE DISJUNTOR PALATINO ENCAPSULADO NA EXPANSÃO RÁPIDA MAXILAR PREVIAMENTE À TERAPIA DE TRAÇÃO REVERSA DA MAXILA: RELATO DE CASO
}

Ricardo Damo Meneguzzi ${ }^{1}$

\section{Resumo}

A expansão rápida maxila (ERM) é uma das principias técnicas de correção da deficiência transversal maxilar, corrigindo, assim a mordida cruzada posterior, além de aumentar o perímetro do arco dentário. Utiliza-se, também, associada à terapia de tração reversa da maxila, por promover a desarticulação das suturas circum-maxilares e favorecer o avanço maxilar por meio de crescimento ósseo aposicional sutural. O presente relato de caso, trata-se da paciente G. R., com 10

Rev Odontol HACO 2019; 1 (1):33-38

\footnotetext{
${ }^{2} \mathrm{MSc}$ em Odontologia,
} $1^{0}$ Tenente QODent ORD HACO; anos de idade, na fase de dentição mista que possui um padrão esquelético de Classe III associado à deficiência maxilar transversa. Além disso, a mesma apresenta hábito de deglutição atípica com interposição lingual, resultando numa mordida aberta anterior. O tratamento proposto foi de ERM, utilizando um disjuntor encapsulado tipo McNamara modificado com grade palatina fixa, seguido de terapia de tração reversa maxilar com máscara facial de Petit. Optou-se pela utilização de um disjuntor palatino encapsulado em virtude da paciente apresentar um plano mandibular com ângulo obtuso, face longa e mordida aberta anterior, cujo deslocamento inferior da maxila, torna-se desfavorável nos pacientes com tendência de crescimento vertical.

PALAVRAS-CHAVE: Técnica de Expansão Palatina; Má Oclusão; Ortodontia Corretiva.

\section{Introdução}

A Ortodontia e a Ortopedia Facial tem por objetivo a correção das desarmonias dentárias e/ou esqueléticas, visando o reestabelecimento das funções estomatognáticas normais, que vão além de uma oclusão favorável, ou seja, busca-se uma harmonia facial, com especial atenção ao perfil mole

Meneguzzi, RD.

Rev Odontol HACO 2019; 1(1):33-38 
e ao arco do sorriso, sem esquecer da mastigação, fonação e respiração ${ }^{1}$.

A disjunção maxilar ou expansão rápida da maxila (ERM), trata-se de um procedimento ortopédico muito empregado na Ortodontia, devido ao seu potencial de correção das desarmonias transversais entre os maxilares, de aumentar o perímetro de arcada, sem alterar desfavoravelmente o perfil facial, e de favorecer a correção do prognatismo mandibular, por meio da tração reversa maxilar, potencializando 0 deslocamento anteroinferior do terço médio da face $\mathrm{f}^{2-4}$. A principal indicação da disjunção maxilar, previamente à tração reversa da maxila, refere-se à desarticulação das suturas circum-maxilares potencializando a resposta das forças protrusivas, bem como a correção da mordida cruzada posterior decorrente da deficiência transversal da maxila ${ }^{3}$. No entanto, a ERM produz um deslocamento inferior da maxila, ocasionando um giro da mandíbula no sentido horário, em relação à base do crânio. Essa rotação mandibular provoca alterações tais como: abertura da mordida, inclinação do plano oclusal, aumento do ângulo do plano mandibular e do eixo $Y$ de crescimento, além do deslocamento do mento para baixo e para trás.

A má oclusão de classe III de Angle pode ser definida como uma desarmonia sagital da face, caracterizada por uma protrusão mandibular, retrusão maxilar ou a combinação de ambas, frequentemente associada a mordida cruzada decorrente de uma atresia transversal da maxila ${ }^{4,5,7,8}$. Alguns tipos de más oclusões podem favorecer o Meneguzzi, RD.

Rev Odontol HACO 2019; 1(1):33-38 surgimento de hábitos bucais deletérios durante o crescimento, incluindo a mordida aberta anterior, podendo gerar alterações significativas dependendo da intensidade, frequência e duração do hábito ${ }^{5}$. COHEN e SILVERMAN ${ }^{7}$, em 1973, preconizaram um disjuntor palatino colado aos dentes superiores para realizar a ERM. As principais vantagens desse aparelho, segundo os autores, são de dispensar a confecção de bandas, facilidade de instalação e promover a intrusão dos dentes posteriores do arco inferior, tendo a melhor indicação para pacientes com tendência de crescimento vertical da face ${ }^{7,9,10}$. Posteriormente, McNAMARA e BRUDON ${ }^{10}$ propuseram modificações no desenho do disjuntor preconizado por COHEN e SILVERMAN ${ }^{7}$, tornando-se o modelo de disjuntor palatino colado mais utilizado, atualmente, em virtude da simplicidade de confecção e bom desempenho clínico. Diante da versatilidade apresentada por esse tipo de disjuntor palatino, o presente relato de caso tem o objetivo de reforçar o seu emprego na prática ortodôntica, especialmente quando o caso atender aos critérios de indicação.

\section{Relato do Caso}

Paciente G. R., leucoderma, gênero feminino, com 10 anos de idade, na fase de dentição mista, apresenta discreto atraso na erupção dos dentes sucessores permanentes. Utilizou chupeta aproximadamente até os 7 anos de idade, substituído pelo hábito de sucção do polegar, resultando numa mordida aberta na região anterior dos arcos, sendo 
perpetuada pela interposição lingual durante o ato de deglutição (Figura 1).

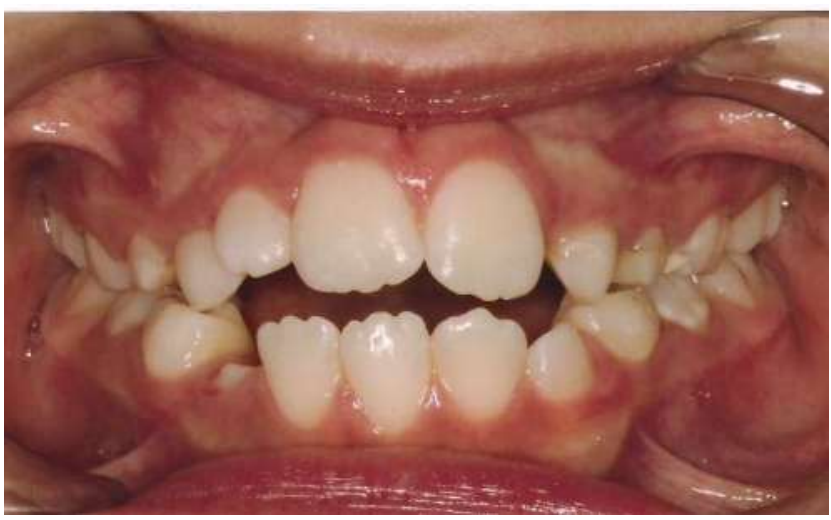

Figura 1 - Vista frontal. Observa-se a atrésia transversal de ambos os arcos, bem como a mordida aberta anterior e a divergência das linhas médias dentárias.

Apresenta um padrão esquelético de Classe III, bem como a relação molar também em distooclusão, sendo compensada pelos tecidos moles que conferem uma aparência agradável, apesar do selamento labial forçado (Figuras $2 \mathrm{~A}, \mathrm{~B}$ ).
Cefalometricamente, observa-se uma tendência de crescimento vertical da face, especialmente por verificar um ângulo mandibular obtuso e plano mandibular aumentado em relação aos valores de referência. A partir da análise de modelos de estudo, verificou-se uma discrepância negativa em ambos as arcadas, decorrente da atresia transversal dos maxilares, gerando, consequentemente, um leve apinhamento na região anterior de ambos os arcos, além de uma não coincidência das linhas médias dentárias. A paciente apresenta um estado de saúde geral muito bom, sem quaisquer alterações sistêmicas. Além disso, o perfil psicológico é colaborador, quesito fundamental para a determinação da conduta terapêutica. Baseando-se nos dados obtidos na anamnese, na consulta clínica e na documentação ortodôntica, elaborou-se um plano de tratamento envolvendo ERM associada à tração maxilar reversa. Em virtude das características acima
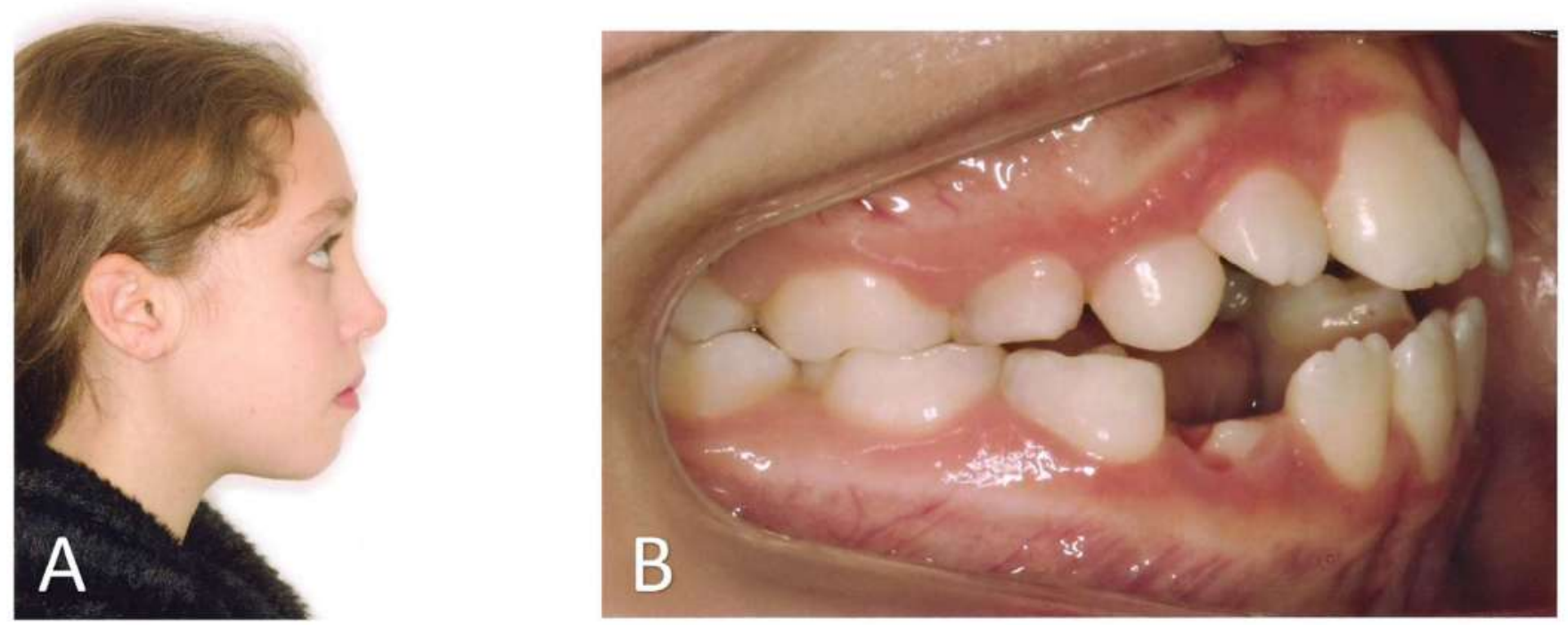

Figura 2 - A: Vista lateral da face. É possível verificar um perfil agradável, apesar da desarmonia esquelética sagital. B: Vista oclusal lateral. É possível verificar a deficiência maxilar no sentido sagital, bem como a mordida aberta anterior e a divergência do plano oclusal. 
citadas do caso, optou-se pelo emprego de um disjuntor palatino encapsulado do tipo McNamara modificado, com a inclusão de grade palatina fixa no mesmo (Figuras $3 \mathrm{~A}, \mathrm{~B}, \mathrm{C}$ ).
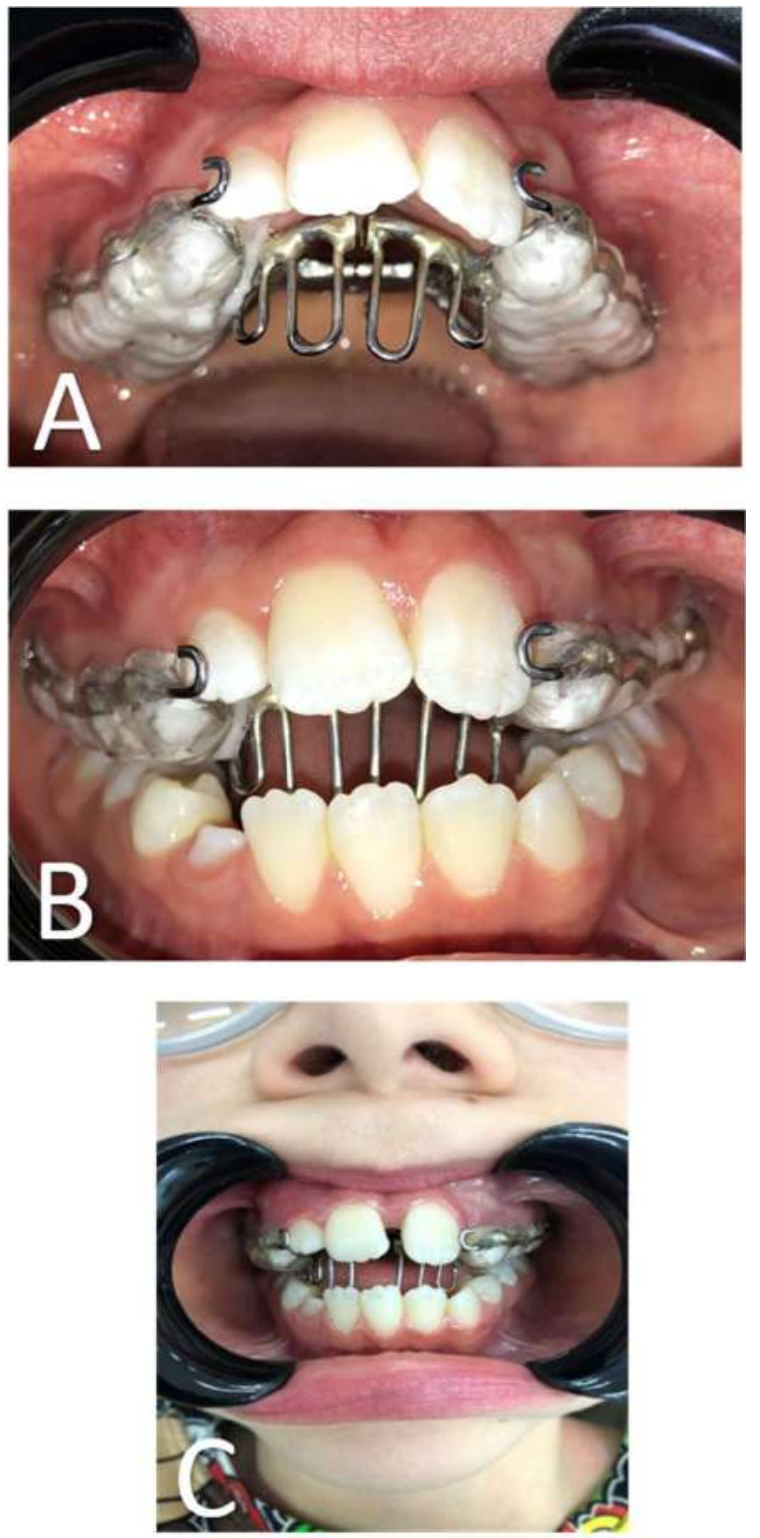

Figura 3 - A,B: Vistas frontal e oclusal, respectivamente, do disjuntor cimentado em boca, após a realização dos ajustes oclusias. C: Vista frontal. Verifica-se abertura da sutura palatina mediana durante o período ativo da ERM.

Após a fase de ativa da ERM, iniciou-se a tração reversa da maxila, por meio de máscara facial Meneguzzi, RD.

Rev Odontol HACO 2019; 1(1):33-38 de Petit (Figura 4), até ser obtida uma sobrecorreção de 3-4 $\mathrm{mm}$ de sobressaliência horizontal, considerando uma possível recidiva da má-oclusão após a suspensão do tratamento ortodôntico. Para a confecção do disjuntor, foi realizada a moldagem de ambas as arcadas com alginato e, em seguida, foi utilizado gesso pedra para vazamento dos moldes, obtendo, assim, os modelos de trabalho. Sobre estes, foram confeccionados, com fio de aço de $1.00 \mathrm{~mm}$ de espessura, o reforço da porção oclusal, circundando os dentes posteriores, os quais foram soldados ao parafuso expansor Hyrax de $9 \mathrm{~mm}$ de ativação. Foram confeccionados, com fio de aço de $0.8 \mathrm{~mm}$ de espessura, a grade palatina e os ganchos para adaptação dos elásticos extra-bucais. Da mesma forma, a grade palatina foi soldada à estrutura do parafuso expansor. Depois de aplicar uma camada de isolante sobre a região dentária do modelo superior, foi confeccionada a cobertura oclusal com resina acrílica autopolimerizável. Após a completa polimerização da porção acrílica, o disjuntor foi removido do modelo de gesso, confeccionadas retenções na região oclusal do mesmo, finalizando o processo com o acabamento e polimento do aparelho. Após a confecção do disjuntor, realizou-se a profilaxia dentária com pedra pomes, seguido de um isolamento relativo da região e, por fim, a cimentação do mesmo com cimento de ionômero de vidro. Foram checados os contatos oclusais e realizados ajustes para distribuir os pontos de contato durante a oclusão habitual e a mastigação. $O$ protocolo de ativação do parafuso expansor foi de 
$1 / 4$ volta pela manhã e $1 / 4$ volta à noite durante 10 dias, sendo o suficiente neste primeiro momento.

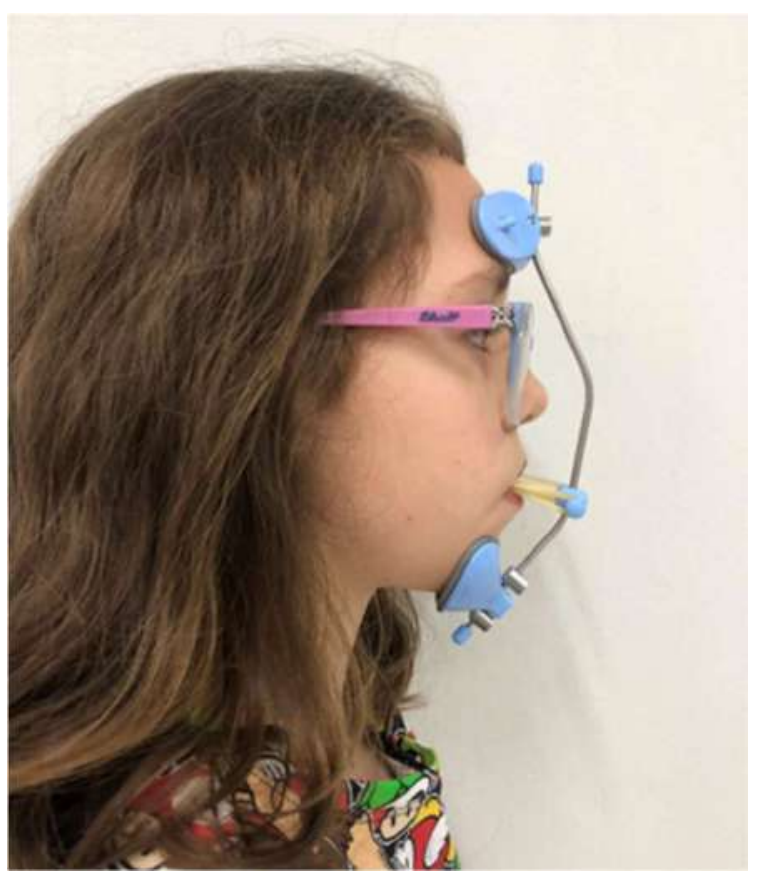

Figura 4 - Vista lateral, observa-se o correto posicionamento da máscara facial de Petit responsável pelo tracionamento maxilar instalada após ERM.

Ao término do período de ativação do disjuntor, o parafuso foi estabilizado com amarrilho metálico e, a partir de então, foi instalada a máscara facial de Petit, dando início à tração reversa da maxila. A paciente também foi encaminhada à Fonoaudiologia para avaliar um possível desequilíbrio da musculatura perioral e da língua, identificando e corrigindo esse fator perpetuador da condição de mordida aberta anterior.

Meneguzzi, RD.

Rev Odontol HACO 2019; 1(1):33-38

\section{Discussão e Conclusão}

A intervenção precoce pode propiciar respostas mais favoráveis, com aparelhos simplificados, devido à grande bioelasticidade óssea nas crianças; redirecionamento dos germes do dentes permanentes para posições mais favoráveis; proporcionar um melhor relacionamento entre as bases ósseas, permitindo o crescimento e o desenvolvimento normais; eliminação das posições inadequadas da ATM, estabelecendo relações simétricas entre o côndilo e a fossa articular; promover uma trajetória mandibular normal; contribuir para a auto-imagem mais favorável da criança, principalmente nos casos onde há comprometimento estético ${ }^{1,11}$. No presente tratamento, esses fatores foram levados em consideração a fim de se obter os melhores resultados com terapia ortopédica/ortodôntica.

O objetivo da disjunção maxilar ou ERM é aumentar transversalmente a arcada dentária superior com o mínimo movimento dos dentes posteriores dentro do processo alveolar. Este procedimento ortopédico envolve a aplicação de forças pesadas aos dentes de suporte e estruturas adjacentes de forma a separar mecanicamente a sutura palatina mediana ${ }^{12-14}$. Os disjuntores bandados, como Haas e Hyrax, são extremamente eficazes para a correção da discrepância transversal, contudo seus efeitos dentários, como extrusão e inclinação dos dentes posteriores e do processo alveolar para vestibular, produzem efeitos negativos 
nos pacientes com padrão de face longa ${ }^{11,13,14}$. Em razão disso, a ERM associada a um batente posterior, oferece ao ortodontista uma forma mais eficiente de disjunção maxilar como menos efeitos colaterais, em pacientes com tendência de crescimento vertical aumentado. Isto se deve à invasão do espaço funcional livre realizado pela cobertura oclusal do aparelho que promove um deslocamento da mandíbula de 2 a $3 \mathrm{~mm}$ abaixo de sua posição de intercuspidação. Consequentemente, os músculos elevadores da mandíbula ficam mais tensionados, comparados a sua posição de repouso, transmitindo, assim, essa tensão aos dentes posteriores e impedindo a extrusão dos mesmos ${ }^{13}$.

Durante a fase de dentição mista, a ERM com disjuntores com batente oclusal pode levar à correção espontânea da má oclusão com tendência de Classe III devido ao discreto avanço mandibular e, mais especificamente, pelo giro mandibular no sentido horário ${ }^{10}$. Com a utilização do disjuntor palatino de McNamara modificado, foi possível obter uma expansão transversal satisfatória, cuja abertura da sutura palatina mediana pode ser constatada clinicamente pelo surgimento de diastema entre os incisivos centrais superiores, o qual foi fechado espontaneamente devido à ação das fibras transseptais. Assim, diante das vantagens que esse tipo de aparelho proporciona, dentre elas, ausência da necessidade de bandas, sua fácil confecção e simplificada instalação, optou-se pela sua utilização no presente relato de caso. Além disso, havia a necessidade de controlar os efeitos verticais Meneguzzi, RD.

Rev Odontol HACO 2019; 1(1):33-38 indesejados devido à presença de mordida aberta anterior, justificando, assim, a presença da cobertura oclusal de acrílico nos dentes posteriores e pela instalação de grade palatina fixa soldada ao disjuntor. A próxima etapa do tratamento, após a ERM, será a tração reversa maxilar por meio da máscara facial de Petit, uma vez que a desarticulação das suturas circum-maxilares proporcionadas pelo procedimento prévio, potencializam o efeito de avanço maxilar e, consequentemente, melhoram a relação esquelética.

A ERM realizada por meio de disjuntor palatino encapsulado, conforme a literatura, tem apresentado resultados clínicos expressivos, especialmente quando bem indicada, como nos casos de atresia maxilar, seja transversal e/ou sagital, e de tendência de crescimento vertical da face, verificada por um ângulo mandibular obtuso e/ou plano oclusal aumentado. Além disso, apresenta-se como uma excelente opção nos casos de dentição decídua ou mista, em que há dificuldade de bandar os dentes posteriores devido à coroa clínica curta.

Conforme o presente relato, a versatilidade do disjuntor palatino encapsulado, pela incorporação de grade palatina fixa e de ganchos para tração reversa da maxila, justifica o emprego desse aparelho na prática ortodôntica.

\section{Referências}

1. DF Siqueira, MS Fernandes, F Cepera, FS Bramante, S Bommarito. Expansor colado simplificado: relato de caso clínico. R Clin Ortodon Dental Press. 2008;7 (1): 52-63. 
2. Almeida RR, Siqueira DF, Bramante FS, Freitas MR, Fuzly A. Aparelho expansor fixo com cobertura de acrílico: técnica de construção e aspectos clínicos. Rev Dental Press Ortodon Ortop Facial. 2000;5(4):62-71.

3. Turley, PK. Orthopedic correction of Class III malocclusion with palatal expansion and custom protraction headgear. J Clin Orthod. 1988;22(5):31425.

4. Capelozza Filho L, Silva Filho OG. Expansão rápida da maxila: considerações gerais e aplicação clínica. Parte I. R Dental Press Ortodon Ortop Facial. 1997;2(3):88-102.

5. Capelozza Filho L, Silva Filho OG. Expansão rápida da maxila: considerações gerais e aplicação clínica. Parte II. R Dental Press Ortodon Ortop Facial. 1997;2(4):86108.

6. Chang $\mathrm{YI}$, Moon SC. Cephlometric evaluation of the anterior open bite treatment. Am J Orthod Dentofacial Orthop. 1999;115(1):29-38.

7. Cohen M, Silverman E. A new and simple palate splitting device. J Clin Orthod.1973;7(6):368-369.

8. Sarver DM, Johnston MW. Skeletal changes in vertical and anterior displacement of the maxilla with bonded rapid palatal expansion appliances. Am J Orthod Dentofacial Orthop. 1989;95(6):462-466.

9. Asanza S, Cisneros GJ, Nieberg LG. Comparison of Hyrax and bonded expansion appliances. Angle Orthod. 1997;67(1):15-22.

10. McNamara JA Jr, Brudon WL. Bonded rapid maxillary expansion appliances. In: Orthodontics and dentofacial orthopedics. 2a ed. Ann Arbor: Needham Press; 1993. p. $145-169$.

11. Silva Filho OG, Valladares Neto J, Rodrigues de Almeida R. Early correction of posterior crossbite: biomechanical characteristics of the appliances. J Pedod. 1989;13(3):195-221.

12. Kiliç $\mathrm{N}$, Kiki A, Oktay H. A comparison of dentoalveolar inclination treated by two palatal expanders. European J of Orthodontics. 2008; 30 (1):67-72.

13. Oliveira DD, Figueiredo DSF, Marinho KC, da Matta Cid Pinto LS, Andrade Jr I. Disjuntor com cobertura oclusal: confecção laboratorial e aplicação clínica. Orthod Sci Pract. 2014; 7(25):92-98.
14. Bishara S.E., Staley R.N. Maxillary expansion: Clinical implications. Am J Orthod Dentofacial Orthop. 1987;91(1):3-14. 